Int. J. Electrochem. Sci., 15 (2020) 4516 - 4533

\title{
Effects of Electrochemical Parameters on the Electropolymerisation of 2-nitro-p-phenylenediamine in Caustic and Neutral Solutions
}

\author{
Mohammad A. Kanan ${ }^{1, *}$, and Ahmad S. Barham ${ }^{2}$ \\ ${ }^{1}$ Industrial Engineering Department, College of Engineering (CE), University of Business and \\ Technology (UBT), Jeddah 21448, Kingdom of Saudi Arabia \\ ${ }^{2}$ General Subjects Department, College of Engineering (CE), University of Business and Technology \\ (UBT), Jeddah 21448, Kingdom of Saudi Arabia \\ *E-mail: m.kanan@ubt.edu.sa
}

doi: $10.20964 / 2020.05 .20$

Received: 4 January 2020 / Accepted: 28 February 2020 / Published: 10 April 2020

\begin{abstract}
A thin film of poly (2-nitro-p-phenylenediamine) (2NPPD) was prepared by electrochemical means of cyclic voltammetric technique. The films were deposited on $1.6 \mathrm{~mm}$ diameter gold electrodes $(A=0.0201$ $\left.\mathrm{cm}^{2}\right)$ or $3.0 \mathrm{~mm}$ glassy carbon $\left(A=0.0707 \mathrm{~cm}^{2}\right)$. We electrochemically investigated 2NPPD monomer in either a caustic solution of $10 \mathrm{mmol} \mathrm{dm}^{-3}$ sodium hydroxide $(\mathrm{pH} 9.2)$ or a neutral solution $(\mathrm{pH} 7.0)$ contained $0.1 \mathrm{~mol} \mathrm{dm}{ }^{-3} \mathrm{KCl}$. A general factorial design was constructed to study the electropolymerisation development of poly 2NPPD by tracking the interactions between the factors of scan rates and the electrode's material types. In the general factorial design, the response was chosen to be the first anodic current peak densities obtained from the CVs in triplicates. In all CV measurements, the rapid drop in the anodic current was highlighted as an indication of electropolymerisation. We obtained good passivation of the poly 2NPPD for the modified electrodes prepared from the neutral medium and caustic aqueous solutions at slower scan rates. At faster scan rates, poor passivation of the modified surfaces was observed, possibly attributed to the thickness of the poly 2NPPD formed and hence the amount of the polymer deposited on the surfaces. Morphological features of poly 2NPPD were inspected using scanning electron microscopy. The diffusion coefficients were measured using the Randles-Sevcik equation and confirmed that the electropolymerisation of 2NPPD monomer is a linear diffusion-controlled process.
\end{abstract}

Keywords: Electropolymerisation; Poly (2-nitro-p-phenylenediamine); General Factorial Design; Electrochemical Cyclic Voltammetry (CV); Diffusion.

$\underline{\text { FULL TEXT }}$ 
(C) 2020 The Authors. Published by ESG (www.electrochemsci.org). This article is an open access article distributed under the terms and conditions of the Creative Commons Attribution license (http://creativecommons.org/licenses/by/4.0/). 